\title{
Vermessen, Klassifizieren, Zuweisen. Das AnKER-Zentrum als machtvolle Organisation der Asylverwaltung
}

\section{Caroline Schmitt}

Online publiziert: 5. Mai 2020

(C) Der/die Autor(en) 2020

Zusammenfassung Der Beitrag untersucht, wie das AnKER-Zentrum als machtvolle Organisation der Asylverwaltung konzeptionell entworfen wird. AnKER ist ein Akronym für „Ankunft, Entscheidung und Rückführung“. Im Mittelpunkt des Beitrags steht eine objektiv-hermeneutische Analyse eines Auszugs aus dem Koalitionsvertrag der Großen Koalition aus dem Frühjahr 2018. Ziel ist eine Rekonstruktion der Mechanismen der Asylverwaltung, die in diesem Dokument zum Ausdruck kommen. Zentrales Ergebnis ist, dass der Koalitionsvertrag das AnKER-Zentrum als Kontrollinstanz konstruiert. Die Unterbringung geht mit einem Zugriff auf Körper, Biografie und Bewegung der Betreffenden einher. Sie werden vermessen, klassifiziert und letztlich ihren Herkunftsländern oder den Kommunen in Deutschland zugewiesen. Mit Michel Foucault lassen sich AnKER-Zentren als Disziplinarinstitutionen fassen. Sie zielen auf ein Einhalten behördlicher Regularien, während sozialarbeiterisches Handeln fall- und lebensweltbezogen ausgerichtet sein soll. Der Beitrag reflektiert auf Basis der Analyseergebnisse die Notwendigkeit Sozialer Arbeit, sich als organisationsbasierte Profession zu begreifen und in die Ausgestaltung von Geflüchtetenunterkünften zu involvieren.

Schlüsselwörter AnKER-Zentrum · Geflüchtetenunterkunft · Asylverwaltung · Flucht · Disziplinarinstitution 


\title{
Measuring, classifying, and assigning. The AnKER center as a powerful organization of asylum administration
}

\begin{abstract}
The article examines how AnKER Center is conceptually designed as a powerful organization of asylum administration. AnKER is an acronym for "arrival, decision and return". The focus of the article is an objective-hermeneutical analysis of an excerpt from the coalition agreement of the Grand Coalition from spring 2018. The aim is to reconstruct the mechanisms of asylum administration, which are expressed in this document. The central result is that the coalition agreement constructs the AnKER Center as a control authority. The accommodation aims to gain access to the people's body, biography, and movements. They are measured, classified, and, in a last step, assigned to their countries of origin or the local town councils in Germany. With Michel Foucault, AnKER Centers can be understood as disciplinary institutions. They aim to comply with official regulations, while Social Work activities should be related to every specific case and people's specific lifeworlds. The article reflects the need for Social Work to understand itself as an organization-based profession and to involve itself in the conception of and discussion on refugee accommodation.
\end{abstract}

Keywords AnKER Center - Refugee accommodation - Asylum administration · Flight $\cdot$ Disciplining institution

\section{Einleitung}

Im Frühjahr 2018 hat die Große Koalition in ihrem Koalitionsvertrag festgehalten, was mittlerweile Lebenswirklichkeit geflüchteter Menschen in den Bundesländern ${ }^{1}$ Bayern, Saarland und Sachsen ist: das Konzept des AnKER-Zentrums. AnKER ist ein Akronym und steht für „Ankunft, Entscheidung und Rückführung“. Anliegen der AnKER-Zentren ist eine Erstunterbringung geflüchteter Menschen in einer Großeinrichtung nach ihrer Ankunft in Deutschland, eine schnelle Bearbeitung von Asylanträgen und Einteilung von Asylantragssteller*innen in Menschen mit ,guter“ und ,schlechter“ Bleibeperspektive (Koalitionsvertrag 2018, S. 107f.). Während erstgenannte auf die Bundesländer, Städte und Kommunen ,verteilt“ werden, sollen letztgenannte abgeschoben oder zu einer ,freiwilligen Rückkehr“ bewogen werden. In Deutschland ist die Unterbringung geflüchteter Menschen auf engem Raum kein Novum. Bereits vor Einführung der AnKER-Zentren war für geflüchtete Familien und Einzelpersonen, für die angenommen wird, dass sie über 18 Jahre alt sind, der Aufenthalt in einer Landeserstaufnahmeeinrichtung (EAE) in allen Bundesländern vorgesehen. Die Bundesländer sind zur Bereitstellung dieser Aufnahmeeinrichtungen verpflichtet ( 44 AsylG). Sie nehmen geflüchtete Menschen nach den Vorgaben

\footnotetext{
1 Die Bundesländer Mecklenburg-Vorpommern und Schleswig-Holstein haben mit dem Bundesinnenministerium die Vereinbarung getroffen, ihre Erstaufnahmeeinrichtungen an die Strukturen von AnKER-Zentren anzugleichen, ohne dass damit zwingend eine Umbenennung einhergeht (Innenministerium Mecklenburg-Vorpommern 2019; Innenministerium Schleswig-Holstein 2019).
} 
des Königsteiner Schlüssels auf, der auf Basis von Einwohner*innenzahl und Steuereinnahmen eines Bundeslandes errechnet, wie viele Menschen ihm zugeteilt werden (§ 45 AsylG). Geflüchtete Menschen werden im Zuge ihres Asylverfahrens hinsichtlich ihres Asyl- und Schutzstatus kategorisiert. Sie verbleiben bis zur Entscheidung des Bundesamtes über den Asylantrag und ggfs. bis zu ihrer Ausreise in den EAEs.

Mit dem Konzept der AnKER-Zentren zielt die Große Koalition auf eine bundesweite Vereinheitlichung der Erstaufnahme (Schader et al. 2018). Das Konzept ist in drei Bundesländern umgesetzt, während die restlichen Länder darauf verweisen, bereits über analoge Organisationsformen zu verfügen oder diese Form der Unterbringung ablehnen (Pürckhauer 2019). Die Debatte um die AnKER-Zentren reiht sich in eine Reihe gesetzlicher Maßnahmen zu einer restriktiven Gestaltung von Fluchtmigrationspolitik ein: So hat der Gesetzgeber die maximale Verweildauer in Unterkünften der Erstunterbringung in den letzten Jahren angehoben. Zu Beginn des Jahres 2020 beläuft sich die Aufenthaltsdauer bei erwachsenen Geflüchteten auf bis zu 18 Monate. Für Eltern oder andere Sorgeberechtigte mit minderjährigen Kindern ist ein maximaler Aufenthalt von bis zu sechs Monaten vorgesehen ( $\$ 47$ AsylG). Geflüchtete Menschen mit abgelehntem Asylantrag und jene, welchen ein Verletzen ihrer Mitwirkungspflichten angelastet wird, können laut „Gesetz zur besseren Durchsetzung der Ausreisepflicht“" bis zu 24 Monate zum Aufenthalt in einer EAE verpflichtet werden. Stimmen aus der Praxis berichten von Fällen, in denen Menschen länger als zwei Jahre in den Einrichtungen verbleiben mussten (Bayrischer Flüchtlingsrat 2019). Nur Personen mit attestierter „Bleibeperspektive“ werden auf die Kommunen verteilt. Die Kommunen handhaben die Folgeunterbringung unterschiedlich. Neben großen Unterkünften stellt eine dezentrale Unterbringung in kleinen Wohneinheiten mancherorts eine Alternative dar (Hinger und Schäfer 2017).

Mit Veröffentlichung des Koalitionsvertrags ging ein Aufschrei durch Nichtregierungsorganisationen, Initiativen und soziale Bewegungen. Es sind vor allem zivilgesellschaftliche Zusammenschlüsse, die über die Situation in den AnKER-Zentren informieren (z. B. Bayerischer Flüchtlingsrat 2019). Angesichts von Berichten über gewalttätige Auseinandersetzungen, fehlende Privatsphäre sowie Isolation regt sich Skepsis, wie Menschenrechte, Kinderrechte und humanitäre Standards in AnKERZentren einzuhalten seien (Hess et al. 2018). Seit Herbst 2018 sind die Zentren in Betrieb, ohne dass die Kritik an ihnen abreißt. Um die Lebenswirklichkeiten der Menschen öffentlich sichtbar zu machen, hat ein Bündnis von Menschenrechtsorganisationen und ehrenamtlichen Unterstützer*innen im Sommer 2019 die MonitoringPlattform anker-watch.de gegründet. Sie berichtet über die Situation in den AnKERZentren und bietet Betroffenen die Möglichkeit, Vorfälle zu melden. Die rechtlichen Verschärfungen im Umgang mit geflüchteten Menschen sind Gegenstand von Stellungnahmen aus dem zivilgesellschaftlichen sowie wissenschaftlichen Bereich (z. B. BAfF 2018; Initiative Hochschullehrender zu Sozialer Arbeit in Gemeinschaftsunterkünften 2016), während empirische Untersuchungen zu und in AnKER-Zentren ein bisher kaum aufgebrochenes Desiderat darstellen. Jedoch liegt ein Korpus an sozial- und kulturwissenschaftlichen Studien zu den Lebenswirklichkeiten geflüchteter Menschen in verschiedenen Formen von Geflüchtetenunterkünften vor. Diese Arbeiten gilt es, in die Analyse von AnKER-Zentren einzubeziehen. Denn wenngleich die spezifische Organisation von AnKER-Zentren neu erscheinen mag, verweist das 
Ansinnen nach Kontrolle geflüchteter Menschen in einer Großunterkunft auf eine historische Kontinuität (Beer 2014).

Die Soziale Arbeit ist von diesen Entwicklungen maßgeblich tangiert und aufgefordert, vor dem Hintergrund rascher Veränderungen in der Asylgesetzgebung eine reflektierte und wissenschaftlich begründete Position zu beziehen und sich gemäß ihres menschenrechtlichen Auftrags zu involvieren. Neben Advocacy Work gemeinsam mit den Betreffenden sind Sozialarbeiter*innen in Geflüchtetenunterkünften präsent und in ihrer Arbeit mit asyl- und ausländerrechtlichen Bestimmungen, den Biografien geflüchteter Menschen und organisationalen Logiken der Geflüchtetenunterbringung konfrontiert. Sie unterstützen unter Bedingungen von Unklarheit über den weiteren Lebensweg. Hierbei agieren sie in einem multiprofessionellen Netzwerk aus Mitarbeitenden der Ausländerbehörden und Sicherheitsdienste, bürgerschaftlich Engagierten, kommunalen Akteur*innen und den Bewohner*innen. Ihr sozialarbeiterischer Auftrag, Teilhabe und Autonomie zu fördern, erscheint im separierten Setting von Geflüchtetenunterkünften als spannungsreiches Unterfangen. So hält etwa Sebastian Muy (2019) fest, dass Sozialarbeiter*innen nicht selten unter Bedingungen agieren, die ihrem menschenrechtlichen Mandat zuwiderlaufen und eine professionelle Aushandlung ihres pädagogischen Auftrags erschweren. Die aufgezeigten Entwicklungen erfordern eine grundlegende Auseinandersetzung Sozialer Arbeit mit der gegenwärtigen Asylpolitik und ihrer Institutionalisierung. Diese Auseinandersetzung kann - so die These des Beitrags - nicht ohne eine Reflexion der organisationalen Mechanismen von Asylverwaltung erfolgen, innerhalb welcher ,über den Verbleib, den Status und die Gestaltung des Aufenthalts von Asylsuchenden [entschieden wird]“ (Lahusen und Schneider 2017, S. 8). Geflüchtetenunterkünfte sind Ausdruck und Element dieser Verwaltungs- und Entscheidungsstruktur. Sie sind genuin nicht-pädagogische Organisationen und zielen auf ein Erfüllen asylrechtlicher Regularien, während sozialarbeiterisches Handeln fall- und lebensweltbezogen ausgerichtet sein soll. Die Konfrontation dieser differenten Logiken verweist auf notwendige „Übersetzungsherausforderungen“ (Engel et al. 2017, S. 4) zwischen Asylverwaltung und pädagogischem Handeln.

Während die Lebenswirklichkeiten in Geflüchtetenunterkünften in Deutschland zunehmend mehr aus der Perspektive geflüchteter Menschen und von Sozialarbeiter*innen erforscht werden (Whistutz 2019; Täubig 2009), findet sich ein organisationsverwalterischer Zugang nur vereinzelt (z. B. Dahlvik 2017; Schittenhelm 2015). Dabei bietet eine solche Perspektive das Potential, Mechanismen von Asylverwaltung $\mathrm{zu}$ entschlüsseln und eine systematische Auseinandersetzung Sozialer Arbeit mit diesen zu ermöglichen. Der vorliegende Beitrag setzt an diesen Überlegungen an und geht der Frage nach, wie das AnKER-Zentrum im Koalitionsvertrag der Großen Koalition aus dem Frühjahr 2018 organisational entworfen wird. Durch eine objektiv-hermeneutische Analyse eines Auszugs aus diesem politischen Dokument werden das Konzept von AnKER-Zentren und die Mechanismen der Asylverwaltung rekonstruiert, die in dieser Konzipierung zum Ausdruck kommen. Von der Konzeptanalyse sind die konkreten Gestaltungs- und Aushandlungsprozesse der Mitarbeitenden, Leitungskräfte und weiterer Instanzen in Geflüchtetenunterkünften methodisch zu unterscheiden. Im Fokus dieses Beitrags steht die Analyse der Konzeptionalisierung auf Basis eines dokumentenbasierten Zugangs mit dem Ziel, in 
einem weiteren Schritt eine sozialarbeiterisch-reflektierte Auseinandersetzung mit den dort entfalteten Organisationsstrukturen anzustoßen.

Der Beitrag widmet sich in Kap. 2 der Historie und wissenschaftlichen Betrachtung von Geflüchtetenunterkünften in Deutschland, bevor Kap. 3 einen Einblick in das methodische Vorgehen der Analyse gibt und die Ergebnisse in Kap. 4 vorgestellt und unter Hinzunahme theoretischer Überlegungen verdichtet werden. Zentrales Ergebnis ist, dass der Koalitionsvertrag das AnKER-Zentrum als Organisation des Vermessens, Klassifizierens und Zuweisens von geflüchteten Menschen konstruiert. Die Organisation verhandelt im Zusammenspiel dieser Mechanismen über eine vermeintlich rechtmäßige Inklusion geflüchteter Menschen in ihr oder Exklusion aus ihrem Zielland Deutschland. Das Dokument zeichnet eine Differenz zwischen geflüchteten Menschen als „Subjekten der Disziplinierung“ und einem diffus bleibenden „Wir“, das als zugehörig zu Deutschland und Kollektiv mit Recht auf Vermessung ,der anderen“ verstanden wird. Kap. 6 diskutiert die Analyseergebnisse in ihrer Bedeutung für die Soziale Arbeit und stellt die Notwendigkeit Sozialer Arbeit heraus, sich als organisationsbasierte Profession zu begreifen und in die konzeptionelle Ausgestaltung von Geflüchtetenunterkünften zu involvieren.

\section{Geflüchtetenunterkünfte in Deutschland. Ein Überblick}

In Deutschland weist die Separation von Menschen mit Flucht- und Migrationserfahrung in segregierten Großeinrichtungen eine historische Kontinuität auf. Nach dem Zweiten Weltkrieg wurden ehemalige Zwangsarbeitslager als Unterkünfte für Vertriebene und Kriegsheimkehrer*innen genutzt (Beer 2014, S. 52 ff.). Mit Anwerbung von Arbeitsmigrant*innen in den 1950er bis 1970er Jahren in Westdeutschland und der Zunahme asylsuchender Menschen in den 1980er und 1990er Jahren manifestierten sich Großunterkünfte als segregierte Orte der Unterbringung für Menschen mit Flucht- und Migrationserfahrung (Muy 2019, S. 191). Die Finanzierung und Organisation der Unterkünfte für Arbeitsmigrant*innen oblag den Unternehmen, in welchen die Menschen ihrer Beschäftigung nachgingen. Die Arbeitgebenden errichteten Barackenlager mit schlechtem Standard, der sich bis Anfang der 1970er Jahre deutlich vom Standard der „Wohnheime für deutsche Arbeitnehmer*innen“ unterschied. Im Laufe der Zeit unterlagen die Unterkünfte schrittweisen Verbesserungen. Von diesen Verbesserungen ausgenommen waren Unterkünfte für asylsuchende Menschen. Diese waren durch fehlende Privatsphäre, starke Reglementierung und die Ausgabe von Sachleistungen statt Geld gekennzeichnet (Dünnwald 2011, S. 8 ff.) - Merkmale, wie sie noch heute in der Unterbringung geflüchteter Menschen virulent sind (Beer 2014). Gegenwärtig erleben Geflüchtetenunterkünfte europa- und weltweit eine „Renaissance“ (Dünnwald 2018; Kreichauf 2018). Sascha Schießl (2018) konstatiert einen gesellschaftspolitischen „Gewöhnungs- und Abstumpfungsprozess“ (Schieß1 2018, S. 16), geflüchtete Menschen in „Einrichtungen einer sich verschärfenden Flüchtlings- und Asylpolitik“ (Schieß1 2018, S. 16) festzusetzen. Die Bandbreite differenter Unterkunftsformate reicht in Deutschland von EAEs über kommunale Gemeinschaftsunterkünfte, Notunterkünfte bis hin zu Abschiebehafteinrichtungen (Christ et al. 2017; Bauer 2017). Die AnKER-Zentren sind im Herbst 2018 als 
weiteres Format hinzugekommen. All diese Einrichtungen erfüllen eine „Scharnierfunktion“ (Schieß1 2018, S. 18) zwischen den dort Untergebrachten und der übrigen Bevölkerung. Innerhalb dieses Rahmens fallen Entscheidungen über einen als legitim klassifizierten Aufenthalt der Menschen in Deutschland oder über ihre Abschiebung. Die sozial- und kulturwissenschaftliche Forschung diskutiert Geflüchtetenunterkünfte unter Rekurs auf differente Konzepte - etwa als ,,totale Institution“ (Täubig 2009) oder „Lager“ (Kreichauf 2018; Thiel und Jahr 2017); dies jedoch nicht ohne Hinweise und empirische Belege dafür, dass geflüchtete Menschen als Akteur*innen mit Fähigkeiten und Ressourcen zu betrachten sind, die selbst innerhalb restriktiver Settings Handlungsmöglichkeiten erschließen (Bochmann 2017). So hält Philipp Schäfer (2015) auf Basis ethnographischer Studien in zwei Leipziger Geflüchtetenunterkünften die Ambivalenz des regulierten Raumes fest: dieser schränkt Teilhabe an der Umgebung ein, gleichzeitig würden aber „Partizipationsflächen" (Schäfer 2015, S. 16) sichtbar. Dies etwa, wenn geflüchtete Menschen jenseits der Unterkunft in Kontakt mit der nicht-geflüchteten Bevölkerung kommen oder sie Einnahmequellen wie das Einsammeln von Pfand erschließen, Markenkleidung erwerben und dem Körper den Anschein gesellschaftlicher Teilhabe verleihen (Schäfer 2015, S. 13f.). Auch der Band von Anne Wihstutz (2019) stellt mit ethnographischen Analysen in drei Berliner Geflüchtetenunterkünften heraus, dass die Rechte von Kindern und ihren Familien gravierend verletzt werden, die jungen Menschen aber kleine Handlungsräume herstellen und etwa das Recht auf Spiel einfordern oder sich gegen einen Mitarbeitenden aus einer Unterkunft mobilisieren. Rebecca Hofmann und Albert Scherr (2017) kommen in ihrer Fallstudie zu einer badenwürttembergischen EAE zu dem Ergebnis, dass sich Mitarbeitende durch hohes Engagement auszeichnen, aber sie die strukturellen Missstände nicht grundlegend beheben können. Sabine Hess und Kolleg*innen (2018) halten in einer Kurzstudie zu den Auswirkungen von AnKER-Zentren fest, dass diese in erster Linie Belastungen bei geflüchteten Menschen hervorrufen und befördern, Nährboden für Vorurteile auf Seite der nicht-geflohenen Gesellschaftsmitglieder sind, internationalen Abkommen, etwa zum Schutz von Frauen und Kindern, nicht gerecht werden, und lokale Unterstützungsstrukturen im Feld der Integration ignorieren, wie sie in der Vergangenheit von Kommunen entwickelt wurden. Sie grenzen Menschen von der übrigen Bevölkerung $\mathrm{ab}$ und setzen sie für einen bestimmten Zeitraum an einem zugewiesenen Ort räumlich fest. Vorliegende Untersuchungen zu Geflüchtetenunterkünften stellen einen Mangel an Privatsphäre, fehlende Rückzugsmöglichkeiten, eine eingeschränkte psychosoziale und gesundheitliche Versorgung, Beratung und Konzentration von Menschen mit unterschiedlichen Biografien, Bedürfnissen und Lebensvorstellungen auf engstem Raum heraus (Dittmer und Lorenz 2016; Dünnwald 2011). Die Alltagswelt der Menschen ist durch Warten, Unsicherheit über die Zukunft und enorme psychosoziale Belastungen gekennzeichnet (Täubig 2009; Pieper 2013). Konflikte und Gewalt werden in einem solchen Umfeld institutionell begünstigt (Krause 2018; Brücker et al. 2016).

Der vorliegende Beitrag schließt an diese Untersuchungen an und wendet sich der Konzipierung von AnKER-Zentren als gegenwärtig kontrovers diskutiertem Eckfall in der Debatte um Geflüchtetenunterkünfte zu. 


\section{Methodisches Vorgehen}

Gegenstand der Analyse ist ein Auszug aus dem Koalitionsvertrag (2018) der Großen Koalition zwischen CDU, CSU und SPD. Das Konzept der AnKER-Zentren ist unter „4. Effizientere Verfahren“ (Koalitionsvertrag 2018, S. 107) auf knapp zwei Seiten ausgeführt. Der Koalitionsvertrag ist ein Dokument, das Informationen zur Organisation der Asylverwaltung in AnKER-Zentren umfasst. Ein solches Dokument fungiert als ,institutionalisierte Spur“ (Wolff 2008, S. 503) eines Verwaltungsapparats und stellt eine ,eigenständige Datenebene“ (Wolff 2008, S. 511) dar. Ziel der Analyse ist eine Rekonstruktion der im Dokument zum Ausdruck gebrachten Strukturlogiken von AnKER-Zentren. Hierbei wird den Empfehlungen Stephan Wolffs (2008) gefolgt, in der „Analyse zunächst von der Selbstgenügsamkeit des Textes“ auszugehen und seine „Eigensinnigkeit“ (Wolff 2008, S. $511 \mathrm{f}$.) zu erschließen, um gründlich in das Textdokument einzutauchen. Hierzu wird auf das sequenzanalytische Vorgehen nach der Objektiven Hermeneutik zurückgegriffen (Oevermann 2002). Eine solche Analyse folgt den Grundprinzipien der wortwörtlichen Interpretation des Materials. Sie basiert auf der gedankenexperimentellen Explikation von Lesarten unter Hinzuziehung sogenannten Welt- und Deutungswissens. Die Bildung von Lesarten orientiert sich am Prinzip der Sparsamkeit. Spezifische Lesarten bestätigen und verdichten sich an immer mehr Stellen des Materials. Zu Beginn der Analyse wird der äußere Kontext, bspw. das Wissen um die Entstehung des Schriftstücks, ausgeklammert und der Fokus wird auf den inneren Kontext gerichtet, d.h. auf den Gehalt des vorliegenden Texts. Ziel objektiv-hermeneutischer Analysen ist die Rekonstruktion latenter Sinngehalte. Im vorliegenden Fall ist damit gemeint, Fallstrukturgesetzlichkeiten, d.h. die grundlegenden Mechanismen in der Konzeption der AnKER-Zentren zu analysieren. Die Objektive Hermeneutik hat ihren Ursprung in der Erforschung innerfamiliärer Interaktionen und der Sozialisationsforschung (Scherf 2009, S. 320). In der Erforschung von Organisationsstrukturen und -prozessen ist sie bisher noch wenig verbreitet (Scherf 2009). Dabei bietet ein objektiv-hermeneutischer Zugang im hier interessierenden Feld die Möglichkeit, durch die Analyse eines politischen Dokuments den Entwurf einer Organisation der Asylverwaltung mit ihren spezifischen Mechanismen zu rekonstruieren.

\section{Ergebnisse}

Die Analyse wird in einem ersten Schritt summarisch dargestellt bei gleichzeitigem Bemühen, die Kleinschrittigkeit im Vorgehen nachvollziehbar zu verdeutlichen (Abschn. 4.1). Im zweiten Schritt werden die Analyseergebnisse verdichtet (Abschn. 4.2). 


\subsection{Analyse des Konzepts AnKER-Zentrum}

Die Ausführungen im ausgewählten Material beginnen folgendermaßen:

4. Effizientere Verfahren (Z. 4988²).

Die Sequenz verweist auf vorausgegangene Gliederungspunkte und ein nach unterschiedlichen Inhalten strukturiertes Werk. An vierter Stelle geht es um Effizienz als Charakteristikum von nicht weiter ausgeführten Verfahren. Effizienz ist ein wirtschaftliches Kriterium zur Beurteilung eines Outputs vor dem Hintergrund der hierzu eingesetzten Mittel: ,„mit geringst möglichem Ressourceneinsatz [...] [soll] ein größtmögliches Ergebnis“ (Büschken 2017, S. 177) erzielt werden. Die angesprochenen Verfahren sollen dabei nicht nur effizient, sondern effizienter als bisher organisiert werden. Die anvisierte Steigerung der Effizienz impliziert eine als unzureichend eingestufte bisherige Praxis. „Verfahren“ verweist auf einen Prozess mit Anfang und Ende, der sich nach spezifischen Regeln gestaltet. Offen bleibt an dieser Stelle, um welche Verfahren es sich handelt und mit welchen Maßnahmen diese optimiert werden können. Die Sequenz lässt mutmaßen, dass sie einem Maßnahmenkatalog entstammt, wie er zur Verbesserung von Abläufen in einem Betrieb verfasst worden sein könnte - denkbar wäre eine Auflistung von Maßnahmen, wie ein Unternehmen die Produktion von Automobilen noch ökonomischer ausrichten könnte. Optimierung, Ökonomisierung und Messbarkeit von Verfahren stehen im Zentrum. Dem schließt sich folgende Passage an:

Menschen, die in Deutschland Schutz suchen, brauchen Asylverfahren, die schnell, umfassend und rechtssicher bearbeitet werden (Z. 4989f.).

Die Sequenz benennt nun das Zielobjekt der Optimierung - es handelt sich nicht um Gegenstände, sondern um „Menschen, die [...] Schutz suchen“. Dies verwundert, insofern die Suche nach Schutz hätte vermuten lassen, dass hierauf mit Hilfe statt mit effizienter zu gestaltenden Verfahren reagiert wird. „Schutz suchen“ bezieht sich auf eine Personengruppe, die einer Gefährdung ausgesetzt ist und sich in Sicherheit bringen will. Ein Angewiesen-Sein auf Dritte kommt zum Ausdruck. Die Sequenz argumentiert ausgehend von einem vermeintlichen Bedarf Schutz suchender Menschen: Ihnen wird zugeschrieben, ein schnelles „Asylverfahren“ zu benötigen.

„Asyl“" bezeichnet einen politisch garantierten Schutz einer Person in einem anderen Land als dem, in dem die Person qua Staatsbürgerschaft zugehörig ist. Die Gewährung von politischem Asyl ist in Deutschland im Grundgesetz, Artikel 16a, festgehalten. „Verfahren“ verdeutlicht, dass für die Gewährung von Asyl ein spezifisches Prozedere durchlaufen werden muss. Entsprechend stellt die Sequenz spezifische Charakteristika heraus, die das „Asylverfahren“ kennzeichnen sollen: „Umfassend" impliziert ein Vorgehen, das alle Teile einschließt. Hiermit verbunden ist ein Aufwand der Recherche. „Umfassend“ und „schnell“" erscheint schwierig zu vereinbaren, insofern eine umfassende Recherche einer gewissen Zeit bedarf. „Rechtssicher" bringt eine weitere Perspektive ins Spiel. Es sind nicht nur die Bedürfnisse der Schutz suchenden Menschen zu berücksichtigen, sondern die Regularien eines

\footnotetext{
2 Die Information in Klammern gibt die Zeilennummer der jeweiligen Passage im Koalitionsvertrag an.
} 
Rechtssystems. „Bearbeitet“ verweist auf einen Verwaltungsapparat zur Abwicklung von Asylverfahren.

Deren Bearbeitung erfolgt künftig in zentralen Aufnahme-, Entscheidungs- und Rückführungseinrichtungen, in denen BAMF, BA, Jugendämter, Justiz, Ausländerbehörden und andere Hand in Hand arbeiten. In den AnKER-Einrichtungen sollen Ankunft, Entscheidung, kommunale Verteilung bzw. Rückführung (AnKER) stattfinden (Z. 4990-4994).

Der wiederholte Hinweis auf die „Bearbeitung“ von Asylanträgen verengt den Blick nun auf den verwalterischen Akt. „Künftig“ weist erneut auf eine intendierte Veränderung einer bisherigen, aber nicht explizierten Praxis hin. Eine Lösung zur Umsetzung der im Vorfeld aufgemachten Anforderungen wird vorgestellt: Die Bearbeitung soll in ,zentralen Aufnahme-, Entscheidungs- und Rückführungseinrichtungen“ erfolgen. „Zentral“ verweist auf ein Zentrum mit weitreichenden Befugnissen. „Aufnahme“, „Entscheidung“ und „Rückführung“ drücken einen chronologischen Ablauf mit der Zieldimension aus, Schutz suchende Menschen dorthin zurückzuführen, woher sie gekommen sind. Interessanterweise fehlen hier Begriffe wie Unterstützung oder Integration. Die Schrittfolge erinnert an eine Maschine - der Ablauf erscheint unabänderlich, abweichende Schritte sind nicht vorgesehen. Der Begriff „AnKER-Einrichtungen“ bringt zum Ausdruck, dass dieser Ablauf in einer Organisation verwaltet werden soll, die durch das Wort „AnKER“ spezifiziert wird. Die Verbindung von Groß- und Kleinschreibung im Wort „AnKER“ verdeutlicht, dass es in der Sequenz nicht im wortwörtlichen Sinne um einen Anker geht. Stattdessen handelt es sich um ein Akronym. Ein Akronym ist ein Kurzwort, das sich aus den Initialen anderer Begriffe bildet und durch die Art des Kürzels spezifische Assoziationen hervorruft; im vorliegenden Fall sind dies Assoziationen an einen Anker. Ein Anker ist eine schwere Kette, die ein Schiff auf den Grund von Meer oder See hinablässt, um an Halt zu gewinnen. Er ist seiner Funktion mehrdeutig und kann ein Schiff stabilisieren und ihm Halt geben, zugleich setzt er das Schiff fest, sodass eine Weiterfahrt nicht möglich ist, ohne den Anker zu lichten. Der Begriff hat zudem metaphorischen Gehalt und wird etwa genutzt, wenn enge Freund*innen bekunden, einander ein Anker in schwierigen Zeiten zu sein. Ein Anker, so die Parallele in beiden Kontexten, gibt Halt in turbulenten Zeiten. In Verbindung mit dem Begriff „Einrichtung“ entsteht in der Sequenz das Bild einer Organisation, die festsetzt, Ruhe ermöglicht und Sicherheit gibt. Zu erwarten gewesen wäre, dass es nun im weiteren Verlauf um die Qualität der Einrichtung geht, also darum, wie sie Schutz suchenden Menschen Sicherheit geben will - etwa durch Personen, die ihnen zuhören und Trost spenden. Diese Lesart muss aber verworfen werden. Denn erneut steht eine verwalterische Funktion im Vordergrund: Die „AnKER-Einrichtungen“ sollen bestehende Instanzen wie Jugendamt oder Ausländerbehörde an einem Ort bündeln. Die Bündelung soll dazu beitragen, dass alle Instanzen „Hand in Hand“ arbeiten. Schutz suchende Menschen erscheinen als Objekte, die von einer zur nächsten Instanz weitergereicht werden. Die Sequenz konkretisiert ein effizient arbeitendes System, das arbeitsteilig die einzelnen Schritte koordiniert und verzahnt. Das Bild einer Maschine drängt sich wieder auf, welche ein Objekt schrittweise bearbeitet. Der Produktionsablauf soll schnell und zweckrational sein und überflüssige Wege 
vermeiden. Alle am Ablauf Beteiligten arbeiten auf dasselbe Ziel hin. Die Schutz suchenden Menschen werden zu Objekten einer Verwaltungsstruktur.

Eine unabhängige und flächendeckende Asylverfahrensberatung ist zu gewährleisten (Z. $4994 \mathrm{f}$.).

Neben der Effizienz wird eine ,unabhängige und flächendeckende Asylverfahrensberatung“ als weiteres Kriterium des Asylverfahrens benannt. Wie diese genau aussieht und von wem sie an welchem Ort angeboten wird, bleibt offen. „Unabhängig“" meint etwas für sich Stehendes, das keiner Instanz unterstellt ist. Hiermit könnte eine Asylverfahrensberatung jenseits der oben genannten Instanzen gemeint sein. „Flächendeckend“ markiert eine räumliche Dimension. Die Beratung soll an jeder Stelle des AnKER-Zentrums zugänglich sein. Es sollen keine Versorgungslücken entstehen. Der Begriff der Beratung untermauert, dass das Asylverfahren nicht selbsterklärend ist, sondern die Betreffenden Informationen brauchen, was auf eine Komplexität des Verfahrens hindeutet. Ein komplexes Verfahren, das in standardisierte Prozesse eingebettet ist, reduziert in erster Linie die Komplexität für den Verwaltungsapparat. Für die zu Beratenden scheint von Bedeutung, Wissen von einer unabhängigen Instanz zu erlangen, um den Prozess überhaupt verstehen zu können. „Gewährleisten“ ruft Assoziationen an die Gewährleistung von Sicherheit, zum Beispiel beim Betreten eines Baugerüsts hervor. Genauso werden spezifische Rechte gewährleistet, zum Beispiel in einem Sozialstaat. Der Begriff umschreibt die tatsächliche Durchsetzung einer Vorschrift oder eines Rechtsanspruchs. Die Beratung soll nicht nur zum Schein existieren. Sie soll dem Papier zu Folge tatsächlich in Anspruch genommen werden können.

Über die Frage von Zuständigkeit und Trägerschaft wird eine Vereinbarung zwischen Bund und Ländern getroffen (Z. 4995 f.).

„Zuständigkeit“" und „Trägerschaft“ können sich entweder auf die Asylverfahrensberatung oder das AnKER-Zentrum insgesamt beziehen. Bund und Länder sollen eine „Vereinbarung“ treffen. Erneut drückt das Papier eine angestrebte Entwicklung aus. Eine Vereinbarung umschließt mindestens zwei Parteien und will Verbindlichkeit herstellen. Die Klärung von Zuständigkeit und Trägerschaft dient der Komplexitätsreduktion und Etablierung dauerhafter Routinen. Hier zeigt sich das Bestreben, nachhaltige Verwaltungsstrukturen zu entwickeln.

Zeile 4997 ist eine Leerzeile. Diese suggeriert, dass nun ein neuer, thematisch eigenständiger Punkt folgt.

Die Bundesrepublik Deutschland hat sich in den vergangenen Jahren in einzigartiger Weise humanitär engagiert. Menschen, die von Krieg und Verfolgung betroffen sind, bieten wir Schutz (Z. 4998-5000).

Die Sequenz wird mit einer nationalstaatlichen Rahmung eröffnet. Es geht um den Staat „Bundesrepublik Deutschland“, dessen humanitärer und außergewöhnlicher Einsatz der vergangenen Jahre herausgestellt wird. Die Betonung von Engagement findet sich zum Beispiel bei Ehrungen und Preisverleihungen für ehrenamtlich Tätige, die sich über das erwartete Maß hinaus in die Gesellschaft einbringen. Analog hierzu hebt die Sequenz das Engagement des Staates Deutschlands hervor und 
bringt zum Ausdruck, dass ein solches Engagement nicht zu den üblichen Aufgaben des benannten Staates gehöre. Das Engagement erscheint als etwas, wofür Dank und Applaus eingefordert werden kann. Dass es sich bei der Gewährung von Schutz unter bestimmten Umständen um einen Rechtsanspruch der Betreffenden handelt, wird nicht genannt. Auf die Feststellung dieses Engagements in der Vergangenheit folgt eine Klärung der Gegenwart: Ein diffus bleibendes ,wir“ bietet spezifischen Menschen ,Schutz“. Schutz wird nicht allen Menschen bedingungslos gewährt, sondern jenen, welche ,,von Krieg und Verfolgung betroffen sind“. „Schutz“ ist auf eine Bedrohung ausgerichtet und meint ein Abschirmen dieser Gefahr. Ein prospektiver Blick ist damit zunächst nicht verbunden. Wie es im Anschluss weitergeht, bleibt offen. Die Passage lässt Bilder von umherwandernden Menschen aufkommen, die bei Regen Schutz in einer Hütte suchen - und diese Hütte mit Besserung der Wetterlage wieder verlassen.

In der Sequenz stehen sich das „Wir“ und die Schutz suchenden Menschen als zwei differente Einheiten in einer durch Abhängigkeit gekennzeichneten Position gegenüber. Das „Wir“ verfügt über die Entscheidungsmacht, ob und wem es Schutz bietet. Diejenigen, die um Schutz bitten, sind dem „Wir“ unterworfen.

Wir haben das Recht zu wissen, wer in unserem Land leben will (Z. 5000).

Im Verlauf des Papiers beansprucht das noch immer diffus bleibende „Wir“ das „Recht“, Wissen über jene einzufordern, welche in „unserem Land“ leben wollen. Das „Wir“ wird als zugehörig zu Deutschland gezeichnet. Diese Zugehörigkeit korrespondiert mit einer Wächterfunktion. „Anderen“ ist ein Aufenthalt in Deutschland nicht bedingungslos möglich. Die Vormachtstellung des „Wir“ wird nicht begründet und erscheint als natürliche und legitime Position. Das Recht, Wissen über Schutz suchende Menschen zu erlangen, drückt den „Preis“ aus, den die Betreffenden für eine mögliche Gewährung von Schutz aus Perspektive des „Wir“ zu zahlen haben. Das Teilen des Wissens bleibt dabei einseitig: Das „Wir“ absorbiert das Wissen über die anderen, ohne selbst ein Wissen über sich preiszugeben. Die Machtasymmetrie zwischen dem „Wir“ und ,den anderen“ wird zementiert. Um welches Wissen es sich handelt und wie es generiert wird, klärt sich im Folgenden:

dazu bestehen besondere Mitwirkungspflichten durch die Ankommenden. Das betrifft zuallererst die umfassende Identitätsfeststellung: Name, Herkunft, Alter, Fingerabdruck. Bei ungeklärter Identität wollen wir die behördlichen Möglichkeiten zu deren Feststellungen erweitern und Identitätstäuschungen wirksamer begegnen. Die umfassende Identitätsfeststellung findet in den AnKEREinrichtungen statt (Z. 5001-5005).

Die Sequenz präsentiert Schutz suchende Menschen im Status der Nicht-Zugehörigen. Um eine ,Eintrittskarte“ in die Bundesrepublik zu erwerben, sind ,besondere Mitwirkungspflichten“ unabdingbar. „Besonders“ verdeutlicht eine Erwartung an die „Ankommenden“, sich zu ,,bemühen“ und aktiv einzubringen. Die Passage erinnert an das Paradigma des Förderns und Forderns eines aktivierenden Sozialstaats, der von seinen Bürger*innen Eigenverantwortlichkeit einfordert und sie aktiviert, Problemlagen selbst zu lösen. Verweigern sie sich diesem Auftrag, hat der Staat die Möglichkeit zur Sanktion, die sich zum Beispiel in einer Kürzung staatlicher 
Unterstützungsleistungen ausdrückt. „Pflichten“ markiert, dass der Appell an Mitwirkung und Eigenverantwortlichkeit nicht nur eine Empfehlung oder einen Wunsch des ,Wir“, sondern eine zu erfüllende Bedingung darstellt. Das Nicht-Erfüllen einer Pflicht wird in der Regel mit Konsequenzen geahndet, die an dieser Stelle unerwähnt bleiben. Die „Mitwirkungspflichten“ beziehen sich „zuallererst“, d.h. in einem ersten, aber nicht hinreichenden Schritt, auf die „umfassende Identitätsfeststellung“. „Umfassend“ taucht hier als Adjektiv erneut auf und wird konkretisiert. Es umfasst die körperliche, biografische und räumliche Vermessung des bewegten Körpers der Menschen. Identität bezieht sich nicht, wie der Begriff vermuten lassen würde, auf subjektive Identifikationen und Empfindungen, sondern auf vermeintlich feststellbare Faktizitäten. Der Verdacht, das „Wir“ könnte im Zuge dieses Prozederes getäuscht werden, wird laut. Täuschungen solle wirksamer als bisher begegnet werden. Sanktionen werden implizit deutlich, aber erneut nicht konkret genannt. Identität - so ließe sich folgern - muss offenbart werden, sonst erfolgt Bestrafung. Der Ort der „Vermessung“ sind die „AnKER-Einrichtungen“.

Die Leerzeile in 5006 kündigt einen von der vorangegangenen Sequenz abgesetzten Gedanken an.

Nach der Altersfeststellung werden unbegleitete Minderjährige durch Jugendbehörden in Obhut genommen, Erwachsene verbleiben in den AnKER-Einrichtungen. Steht in Zweifel, ob es sich um Jugendliche oder um Erwachsene handelt, erfolgt die Altersfeststellung durch das zuständige Jugendamt unter Beteiligung des BAMF in den AnKER-Einrichtungen (Z. 5007-5011).

Die Sequenz erklärt das Prozedere, das auf die Identitätsklärung folgt. Die „Altersfeststellung“ erscheint hierbei als entscheidender Wegweiser: Unbegleitete Minderjährige sollen durch die Jugendbehörden in Obhut genommen und aus den Einrichtungen entfernt werden. Die Einrichtungen scheinen als nicht kind- und jugendgerecht für unbegleitete Minderjährige eingestuft zu werden. Für Erwachsene erachtet das „Wir“ die Einrichtungen als ,,adäquaten Ort“, der für ihren Verbleib vorgesehen ist. Angestrebt wird eine eindeutige Klassifikation: entweder minderjährig oder erwachsen. Kann diese Unterscheidung nicht vorgenommen werden, sollen Jugendamt und BAMF unterstützen. Die konkrete Vorgehensweise bleibt unbenannt. Im Fokus steht erneut die Verzahnung der in die einzelnen Verfahrensschritte involvierten Instanzen. Sie sollen das Verfahren legitim erscheinen lassen und ein sachund fachgerechtes Agieren suggerieren. Weiter heißt es:

Um die Chance auf eine erfolgreiche Integration zu wahren und europarechtliche Vorgaben zu erfüllen, ist die Bleibeverpflichtung in den AnKER-Einrichtungen zeitlich und sachlich zu begrenzen. Sowohl in den Aufnahmeeinrichtungen als auch in den AnKER-Einrichtungen soll die Aufenthaltszeit in der Regel 18 Monate nicht überschreiten ( $\$ 47$ Abs. 1a und 1b Asylgesetz bleibt davon unberührt), bei Familien mit minderjährigen Kindern in der Regel sechs Monate. Insgesamt ist eine geschlechter- und jugendgerechte Unterbringung zu gewährleisten (Z. 5013-5019).

Die Sequenz hebt den Faktor Zeit als Qualitätsmerkmal hervor, um Integrationschancen nicht zu blockieren und ,europarechtliche Vorgaben“ zu erfüllen. Die 
Betonung dieser beiden Aspekte drückt aus, dass Integration und europarechtliche Vorgaben potentiell durch die AnKER-Einrichtungen untergraben werden könnten. Um dies zu verhindern, wird der Aufenthalt in den ,Einrichtungen“ zeitlich begrenzt; so würden Integrationschancen und europarechtliche Bestimmungen noch gewährleistet. Die Sequenz formuliert nicht, wie Integration und eine Umsetzung der nicht näher spezifizierten Bestimmungen gezielt erfüllt werden könnten, sondern ex negativo, wie erreicht werden kann, dass diese nicht verletzt werden. Das Herausstellen einer „Bleibeverpflichtung“ konkretisiert einen machtvollen Zugriff auf die räumliche Bewegung von Menschen. Der Ort des Aufenthalts wird mit den AnKER-Einrichtungen vorgeschrieben und ist nicht frei wählbar. Auch die zeitliche Dimension des Aufenthalts ist fremdbestimmt; für Familien mit Kindern wird die zeitliche Dauer nach unten korrigiert. Innerhalb des Settings sollen diffus bleibende geschlechter- und jugendgerechte Vorkehrungen getroffen werden.

Eine Festsetzung von Menschen an einem bestimmten Ort für eine von anderen festgeschriebene Dauer erfolgt üblicherweise in einem Gefängnis. Die Schutz suchenden Menschen erscheinen in der Sequenz als Insass*innen. Sie warten auf ihren Urteilsspruch und sind abhängig von einer Wächterinstanz. Statt Sicherheit dominiert eine Kontrolllogik. Die weitere Sequenz konkretisiert das von dieser Instanz auszusprechende „Urteil“":

Wir streben an, nur diejenigen auf die Kommunen zu verteilen, bei denen eine positive Bleibeprognose besteht. Alle anderen sollen, wenn in angemessener Zeit möglich, aus diesen Einrichtungen in ihre Heimatländer zurückgeführt werden (Z. 5021-5023).

Das „Wir“ attestiert auf Basis des gewonnenen Wissens das Urteil: eine positive oder negative „Bleibeprognose“. Eine Prognose richtet sich auf einen zukünftig voraussichtlich eintretenden Zustand und stützt sich auf Methoden, mit denen sie bestimmt werden kann. Sie wird üblicherweise von einer Person mit Expert*innenstatus in einem spezifischen Fachgebiet ausgestellt. Die Bezeichnung „Prognose“ lenkt den Blick weg davon, dass das Bleiben-Können oder Gehen-Müssen kontroversen politischen Aushandlungen unterliegt und suggeriert, sich auf nicht verhandelbare, nicht beeinflussbare Kriterien zu stützen. Die Verteilung von Menschen mit guter Bleibeprognose auf die Kommunen macht Schutz suchende Menschen zu fremdgesteuerten Objekten in einem nicht selbst wählbaren Raum. Das Prozedere ist dabei in mehrere Schritte untergliedert: die Menschen werden zunächst vermessen (Wer und wie alt sind sie?), dann wird eine Prognose erstellt, welche die Menschen klassifiziert (,gute“ vs. „schlechte“ Bleibeprognose). Und schließlich werden sie ihres vermeintlich rechtmäßigen Platzes zugewiesen. Das Verfahren ist eine Maschine, die feststellt, sortiert und platziert. Die Platzierungspraxis reicht dabei über den Ort des AnKER-Zentrums hinaus. Sie ist in ihrer Wirkmacht territorial entgrenzt. Menschen mit schlechter „Bleibeprognose“ werden von der Maschinerie nach Möglichkeit in ihre „Heimatländer“ zurückgeführt. Die Bezeichnung „Heimatland“ nimmt dabei eine Platzierung der als nicht-zugehörig Kategorisierten durch die Maschinerie im AnKER-Zentrum vor. Doch nicht nur die räumliche, sondern auch die zeitliche Wirkmacht erstreckt sich über den Ort der Einrichtung hinaus: 
Spätestens drei Jahre nach einer positiven Entscheidung ist eine Überprüfung des gewährten Schutzes erforderlich. Für dieses Prüfverfahren werden verbindliche Mitwirkungspflichten der Betroffenen gelten. Dazu sollen Belehrungen stattfinden (Z. 5025-5027).

Über mehr als drei Jahre hinweg unterliegen schutzsuchende Menschen mit „,positiver Entscheidung“ dem Eingriff des „Wir“. Sie sind aufgefordert, ihren Aufenthalt in zeitlichem Abstand zu legitimieren und erneut überprüfbar zu machen. „Schutz“ wird als befristeter Bedarf konstruiert und damit als etwas, das Menschen zu einem bestimmten Zeitpunkt legitim beanspruchen, aber auch wieder verlieren können. In letztem Fall kann ein Entzug des Schutzes durch das „Wir“ erfolgen. Erneut werden die „Mitwirkungspflichten“ der Schutz suchenden Menschen herausgestellt. Diese sind nicht verhandelbar, sondern verbindlich. „Belehrungen“ zu den Mitwirkungspflichten sollen derart auf die Menschen einwirken, dass kein Widerstand zu erwarten ist. Die Einwirkungen erscheinen als Züchtigung und verdeutlichen eine Differenz zwischen jenen, die belehren und mit Befugnissen zur Sanktion ausgestattet sind, und jenen, welche belehrt werden und gehorchen sollen. Diese Binarität erinnert an eine Richter*in-Gerichteten-Konstellation, die den Gerichteten nach dem Urteilsspruch wenig bis keinen Handlungsspielraum lässt.

\subsection{Vermessen, Klassifizieren, Zuweisen. Die organisationalen Mechanismen des Konzepts AnKER-Zentrum}

Die Analyse verdeutlicht, dass das AnKER-Zentrum im Dokument als machtvoller Apparat entworfen wird, der systematisch auf Körper, Biografie und Bewegung der betreffenden Menschen Zugriff erlangen will. Das Konzept zeichnet sich durch die Institutionalisierung eines standardisierten Mechanismus des Vermessens, Klassifizierens und Zuweisens aus. Die Vermessung meint das Einholen von Daten über Biografie, Körper und bisherige Mobilität. Dem folgt die Klassifikation, ob sich die Suche nach Schutz auf Gründe stützt, welche von der machtvollen Apparatur als legitim oder illegitim eingestuft werden. Die Maschinerie des AnKER-Zentrums suggeriert, sich in der Klassifikation auf ein „objektives“ Wissen zu stützen, ohne dessen Legitimität unter Beweis zu stellen. Es wird eine Prognose ausgestellt, welche zwischen Menschen mit „guter“ und „schlechter Bleibeperspektive“ unterscheidet. Die Zuweisung auf die Kommunen soll nur für erstgenannte erfolgen. Letztgenannte gilt es bei negativ beschiedenem Asylantrag in die Herkunftsländer abzuschieben. Die Wirkmacht des AnKER-Zentrums reicht damit über den konkreten Ort hinaus. Es steuert eine zukünftige Bewegung, während es die Menschen zum Zeitpunkt des Aufenthalts immobilisiert. Die Menschen sind einem zweckrationalen, auf ökonomischen Prinzipien beruhenden Verwaltungsapparat unterworfen, der soziale Zugehörigkeiten und räumliche Zuweisungen bestimmen und durchsetzen will. Paradoxerweise werden geflüchtete Menschen zu einer Mitwirkung an ihrer Kontrolle aufgefordert: Sie selbst sind es, die Daten über sich liefern und der Kontrollinstanz die Mittel zu ihrer Beherrschung darbieten sollen. Der in machtvolle Abhängigkeitsstrukturen eingebettete Appell zur unbedingten Mitwirkung am Prozess des Vermessens ist Ausdruck von Disziplinierung, die dem Subjekt als „Eintrittskarte“ in ein Verfahren 
dient, das sich in allen Verfahrensschritten einer Partizipation durch die Betreffenden entledigt. Die disziplinierenden Mechanismen des AnKER-Zentrums lassen sich mit Michel Foucaults (2019 [1976]) Überlegungen zur Disziplinarinstitution fassen und zeigen die Aktualität seiner Ausführungen für Geflüchtetenunterkünfte auf (Rölli und Nigro 2017). Das AnKER-Zentrum verteilt Individuen im Raum und operiert im Modus des Parzellierens und Zuordnens der angeordneten Elemente (Foucault 2019 [1976], S. 183 ff.). Dabei kotrolliert es nicht nur den Ort, an dem sich ein Individuum aufhält, sondern entfaltet zugleich ein Zeitregime. Es zerlegt Handlungen von Körpern in Einheiten, gliedert die Zeit in einzelne Segmente mit spezifischen Funktionen und stellt einen ,leistungsfähigen Apparat“ (Foucault 2019 [1976], S. 212) her, der einen machtvollen Zugriff auf menschliche Körper ausübt. Der einzelne Körper ist eingebunden in die Platzierung anderer Elemente und wird zum Objekt disziplinarischer Abläufe. „Architektur, Funktionen und Hierarchien“ (Foucault 2019 [1976], S. 190) bilden in ihrer Gesamtheit ein räumlich und zeitlich strukturiertes „Befehlssystem“ (Foucault 2019 [1976], S. 214).

\section{Soziale Arbeit und Organisationen der Asylverwaltung. Herausforderungen und Perspektiven}

Die Analyse hat die konzeptionell angelegte Unterwerfung von Menschen in Organisationen der Asylverwaltung aufgezeigt. Die AnKER-Zentren werden im Koalitionsvertrag als Orte erzwungener und verwalteter Immobilität in einer durch Mobilität gekennzeichneten Welt entworfen. Während Menschen, Dinge und Ideen im ,Zeitalter der Bewegung“ (Nail 2019) in bis dato nicht da gewesener Regelmäßig- und Schnelligkeit potentiell große Distanzen zurücklegen können, sind AnKER-Zentren Ausdruck von Mobilitätsregimen, die Bewegungen steuern, gezielt verhindern und soziale Ungleichheit produzieren und verfestigen (Salazar 2018). Einer konstatierten Normalität von Mobilität stehen sie als Orte erzwungener Immobilität kontrastiv gegenüber und lassen globale ethische Probleme deutlich hervortreten (Jensen 2009): Wer darf darüber entscheiden, wer sich wann und für wie lange wohin bewegt und dort aufhält? Die Analyse hat herausgestellt, dass AnKER-Zentren diese Bestimmungsmacht für sich beanspruchen, sie in ein Verwaltungsprozedere übersetzen und die Bewegung geflüchteter Menschen durch die institutionalisierten Mechanismen des Vermessens, Klassifizierens und Zuweisens steuern und begrenzen wollen. Geflüchtete Menschen verlieren im Zuge dieses Prozesses an Subjektivität und sind als fremdbestimmte Masse einer auf ökonomischen Prinzipien aufbauenden Verwaltungsapparatur unterworfen. Diese Apparatur korrespondiert mit Foucaults Konzipierung der Disziplinarinstitution (2019 [1976]), deren Funktion er am Beispiel der Kaserne expliziert. Die Unterbringung umherziehender Soldaten sei für die Bevölkerung schlecht zu ertragen, sie müsse ,beruhigt werden“ (Foucault 2019 [1976], S. 182). Der segregierte Raum der Kaserne verspreche für das „Außen“ Kontrolle und verhindere eine als beängstigt erlebte Mobilität der Soldatengruppe. Die Disziplinarinstitution hat damit hohe symbolische Bedeutung und ist Ausdruck des Versuchs, Ordnung im vermeintlichen Chaos herzustellen. Im Fall von AnKERZentren kann die These aufgestellt werden, dass durch Segregation, Disziplinierung 
und Kontrolle geflüchteter Menschen auf engstem Raum die Illusion zu erhalten versucht wird, die „Oberhand“ darüber zu bewahren, wann und wie sich geflüchtete Menschen fortbewegen. Geflüchtete Menschen werden als „unerwünschte Masse“ und ,potentiell bedrohliche andere“ gezeichnet, vor welchen die nicht-geflüchtete Bevölkerung geschützt werden muss. Die Figur des Schutzes, welche der Koalitionsvertrag zunächst als Bedürfnis geflüchteter Menschen herausstellt, kehrt sich letztlich um: Es ist das „Wir“ der nicht-geflüchteten Mehrheitsgesellschaft, das sich mit der Organisation der AnKER-Zentren vor der als gesichtslos konstruierten Masse geflüchteter Menschen dadurch schützen will, dass es sie von sich abschirmt.

Befähigung, soziale Unterstützung und Anwaltschaft, wie sie als Prinzipien in der Sozialen Arbeit verwurzelt sind, stehen konträr zu den organisationalen Mechanismen der Mobilitätsbegrenzung und Fremdbestimmung. Sozialarbeiter*innen und Erzieher*innen sind in den AnKER-Zentren präsent, wenn die Jugendämter involviert oder Formen der Kinderbetreuung und Sozialberatung in den Zentren realisiert werden. Sie sind mit einer Organisationsstruktur konfrontiert, die pädagogischen Prinzipen zuwiderläuft. Wie ist professionelles Handeln unter diesen Bedingungen möglich? Und aus Perspektive geflüchteter Menschen gefragt: Wie ist Überleben und Alltagsleben in einem solchen Setting bewältig- und gestaltbar?

Die Konzeptanalyse verdeutlicht die zweckoptimierte Stoßrichtung des AnKERZentrums. Es ist ausgerichtet auf eine ökonomische Verwaltung und Kontrolle von Menschen mit komplexen Biografien und Erfahrungen. Empirische Studien weisen darauf hin, dass geflüchtete Menschen Zeit, vertrauensvolle und routinierte Beziehungen und sichere Orte brauchen, um über ihre Erfahrungen im Herkunftsland und auf der Flucht zu sprechen (z.B. Zeller et al. 2020). Ihre Konfrontation mit einer derart konzipierten Organisation lässt die These $\mathrm{zu}$, dass sich unter diesen Bedingungen nur schwer pädagogische Beziehungen aufbauen lassen. In diesem Zusammenhang ist unabdingbar, dass sich Soziale Arbeit als organisationsbasierte Profession versteht und sich in die Ausgestaltung der Geflüchtetenunterbringung involviert, um die Bedingungen für ihr pädagogisches Wirken überhaupt zu schaffen. Soziale Arbeit ist grundlegend von bürokratischen Prozessen und (nicht-)pädagogischen Organisationen umspannt und in deren Herstellung selbst involviert (Schröer und Wolff 2018). Die Gestaltung von Organisationen ist eine ihrer genuinen Aufgaben und verbindet „System- und Akteursperspektive“ (Göhlich und Tippelt 2008, S. 633). Im Zuge disziplinierender Organisationen der Asylverwaltung ist eine aktive Mitwirkung unabdingbar. So gilt es, innerhalb von Geflüchtetenunterkünften Öffnungen zu erschließen, multiprofessionell mit anderen Instanzen in Austausch $\mathrm{zu}$ treten und die Unterkünfte trotz aller Barrieren und Restriktionen als potentiell veränderbare „Sozialgebilde“ (Göhlich et al. 2014, S. 4) zu begreifen. Hiermit entlastet sich die Soziale Arbeit nicht von einem Advocacy Work auf politischer Ebene und einer grundlegenden Kritik an der Segregation geflüchteter Menschen in Großunterkünften; da jedoch von einem längerfristigen Überdauern dieser Strukturen auszugehen ist, bedarf es einer Einmischung in die Asylverwaltung, ohne diese zu normalisieren (Hofmann und Scherr 2017, S. 12). Die gegenwärtigen Debatten um Gewaltschutz, Mindeststandards und Beschwerdemanagement in Geflüchtetenunterkünften bieten hierzu Ansatzpunkte. Im Jahr 2016 wurde von UNICEF zusammen mit dem Bundesfamilienministerium die Initiative „Schutz von geflüchteten 
Menschen in Flüchtlingsunterkünften“" mit dem Ziel einer bundesweiten Förderung von Schutzkonzepten ins Leben gerufen. In Sachsen hat der Sächsische Ausländerbeauftragte den sogenannten Heim-TÜV (2017) institutionalisiert, der das Ziel verfolgt, die Lebensbedingungen von Asylsuchenden in Geflüchtetenunterkünften in Sachsen zu verbessern. Diese Debatten bieten Potential, Entwicklung und Erforschung solcher Konzepte mit zu steuern (Enders 2018). Sie offerieren Anschlusspunkte an Diskurse zu Inklusion (Homfeldt 2020) und zu Gewaltschutz, wie sie in der Sozialen Arbeit gegenwärtig z.B. in der Pflege (Horn und Schweppe 2019) oder in Bezug auf den Schutz vor sexueller Gewalt in pädagogischen Einrichtungen (Retkowski et al. 2018) verhandelt werden. An dieser Stelle wird deutlich, dass die Konzipierung organisationaler Instrumente grundlegende Fragen Sozialer Arbeit berührt. Ausschließende Organisationen der Asylverwaltung sind Ausdruck restriktiver Asylpolitik und kontroverser Debatten. Eine sich involvierende Soziale Arbeit macht diese Form der Unterbringung zum Gegenstand der Diskussion. Dazu bedarf es einer Analyse nicht nur der asylverwaltenden Großunterkünfte, sondern ebenso alternativer inklusiver und solidarischer Formen des Wohnens und Zusammenlebens, wie sie vielerorts bereits praktiziert werden. Beispiele hierfür sind das Sharehouse Refugio, Berlin oder das Grandhotel Cosmopolis, Augsburg. Projekte wie diese können als Ausdruck eines zivilgesellschaftlichen Strebens nach solidarischen Städten, „urban citizenship“ (Kewes 2016) und Widerstands gegen nationalgesellschaftliche Abschottungstendenzen gegenüber Menschen auf der Flucht gelesen werden (Loick 2017, S. 13). Eine Reflexion von Geflüchtetenunterkünften und ihrer Alternativen bietet Perspektiven, gemeinsam mit den Betreffenden neue Möglichkeiten zum Umgang mit Fluchtmigration aus der Profession und Disziplin Sozialer Arbeit heraus zu entwickeln.

Funding Open Access funding provided by Projekt DEAL.

Open Access Dieser Artikel wird unter der Creative Commons Namensnennung 4.0 International Lizenz veröffentlicht, welche die Nutzung, Vervielfältigung, Bearbeitung, Verbreitung und Wiedergabe in jeglichem Medium und Format erlaubt, sofern Sie den/die ursprünglichen Autor(en) und die Quelle ordnungsgemäß nennen, einen Link zur Creative Commons Lizenz beifügen und angeben, ob Änderungen vorgenommen wurden.

Die in diesem Artikel enthaltenen Bilder und sonstiges Drittmaterial unterliegen ebenfalls der genannten Creative Commons Lizenz, sofern sich aus der Abbildungslegende nichts anderes ergibt. Sofern das betreffende Material nicht unter der genannten Creative Commons Lizenz steht und die betreffende Handlung nicht nach gesetzlichen Vorschriften erlaubt ist, ist für die oben aufgeführten Weiterverwendungen des Materials die Einwilligung des jeweiligen Rechteinhabers einzuholen.

Weitere Details zur Lizenz entnehmen Sie bitte der Lizenzinformation auf http://creativecommons.org/ licenses/by/4.0/deed.de.

\section{Literatur}

Bauer, I. (2017). Unterbringung von Flüchtlingen in deutschen Kommunen. Konfliktmediation und lokale Beteiligung. State-of-Research Papier 10. https://flucht-forschung-transfer.de/wp-content/uploads/ 2017/05/IB-SoR-10-BAUER_Konfliktmediation-1.pdf. Zugegriffen: 21. Febr. 2020. 
Bayerischer Flüchtlingsrat (2019). Schriftliche Stellungnahme zur Expert*innenanhörungzum Thema „AnkER-Einrichtungen in Bayern“. https://www.fluechtlingsrat-bayern.de/tl_files/2019/Dokumente/ Statement\%20Frau\%20Grote.pdf. Zugegriffen: 21. Febr. 2020.

Beer, M. (2014). Die deutsche Nachkriegszeit als Lagergeschichte. Zur Funktion von Flüchtlingslagern im Prozess der Eingliederung. In H. Bispinck \& K. Hochmuth (Hrsg.), Flüchtlingslager im Nachkriegsdeutschland (S. 47-71). Berlin: Ch. Links.

Bochmann, A. (2017). Soziale Institution Lager. In S. Lessenich (Hrsg.), Geschlossene Gesellschaften. Verhandlungen des 38. Kongresses der Deutschen Gesellschaft für Soziologie in Bamberg 2016 (S. 1-9). http://publikationen.soziologie.de/index.php/kongressband_2016/article/view/480. Zugegriffen: 12. Aug. 2019.

Brücker, H., Rother, N., \& Schupp, J. (2016). IAB-BAMF-SOEP-Befragung von Geflüchteten. Deutsches Institut für Wirtschaftsforschung Berlin. https:/www.econstor.eu/handle/10419/149124. Zugegriffen: 21. Febr. 2020.

Bundesweite Arbeitsgemeinschaft der Psychosozialen Zentren für Flüchtlinge und Folteropfer (BAfF) (2018). Abschottung um jeden Preis? Wie sich die geplanten Ankerzentren auf Geflüchtete, Traumatisierte und die Gesellschaft auswirken. www.baff-zentren.org/wp-content/uploads/2018/06/ Stellungnahme_BAfF_Ankerzentren_2018.06.18.pdf. Zugegriffen: 20. Febr. 2020.

Büschken, M. (2017). Soziale Arbeit unter den Bedingungen des „,aktivierenden Sozialstaates“. Weinheim und Basel: Beltz.

Christ, S., Meininghaus, E., \& Röing, T. (2017). „All Day Waiting“. Konflikte in Unterkünften für Geflüchtete in NRW. Bonn International Center for Conversion (BICC). https://www.bicc.de/uploads/ tx_bicctools/BICC_WP_3_2017_web_01.pdf. Zugegriffen: 21. Febr. 2020.

Dahlvik, J. (2017). Entscheiden über Asyl. Organisationssoziologische Überlegungen zum Zusammenspiel von Formalität und Informalität im österreichischen Asyl-Verwaltungsverfahren. In C. Lahusen \& S. Schneider (Hrsg.), Asyl verwalten. Zur bürokratischen Bearbeitung eines gesellschaftlichen Problems (S. 117-143). Bielefeld: transcript.

Dittmer, C., \& Lorenz, D.F. (2016). Waiting for the bus that never comes. https://refubium.fu-berlin. de/bitstream/handle/fub188/21907/KFS_Working_Paper_3_Dittmer_Lorenz_Waiting_for_the_bus_ that_never_comes.pdf?sequence=1\&isAllowed=y. Zugegriffen: 21. Febr. 2020.

Dünnwald, S. (2011). Die Bundesrepublik als Lagergesellschaft. In Landesflüchtlingsräte (Hrsg.), AusgeLAGERt. Zur Unterbringung von Flüchtlingen in Deutschland (S. 8-12). https://www.frsh.de/ fileadmin/schlepper/schl_54/s54_8-12.pdf. Zugegriffen: 8. Aug. 2019.

Dünnwald, S. (2018). Die Renaissance der Lager. Die Ankerzentren setzen eine deutsche Tradition der Ausgrenzung fort und rufen deren Ursprünge direkt wieder auf. ak-analyse \& kritik, 640, o.S.

Enders, U. (2018). Kinderrechte und Beschwerdemanagement in Flüchtlingsunterkünften. In L. Hartwig, G. Mennen \& C. Schrapper (Hrsg.), Handbuch Soziale Arbeit mit geflüchteten Kindern und Familien (S. 616-621). Weinheim und Basel: Beltz Juventa.

Engel, N., Göhlich, M., Schröer, A., \& Weber, S. M. (2017). Spezifische Organisationen als Orte organisationspädagogischer Forschung und Praxis: Eine Einführung. In M. Göhlich, A. Schröer \& S. M. Weber (Hrsg.), Handbuch Organisationspädagogik (S. 1-9). Wiesbaden: VS.

Foucault, M. (2019 [1976]). Überwachen und Strafen. Die Geburt des Gefängnisses (17. Aufl.). Frankfurt am Main: Suhrkamp.

Göhlich, M., \& Tippelt, R. (2008). Pädagogische Organisationsforschung. Einführung in den Thementeil. Zeitschrift für Pädagogik, 54(5), 633-636.

Göhlich, M., Weber, S.M., \& Schröer, A. (2014). Forschungsmemorandum Organisationspädagogik. Kommission Organisationspädagogik der DGfE. https://www.dgfe.de/fileadmin/OrdnerRedakteure/ Sektionen/Sek14_OrgaPaed/2014_Forschungsmemorandum_Organisationspädagogik.pdf.. Zugegriffen: 20. Febr. 2020.

Heim-TÜV (2017). Evaluation der dezentralen Unterbringung und der unteren Ausländerbehörden im Freistaat Sachsen. https://sab.landtag.sachsen.de/dokumente/landtagskurier/SAB-HEIM_A4-TUEVinteraktiv-2017.pdf. Zugegriffen: 21. Febr. 2020.

Hess, S., Pott, A., Schammann, H., Scherr, A., \& Schiffauer, W. (2018). Welche Auswirkungen haben „Anker-Zentren“? Eine Kurzstudie für den Mediendienst Integration. Berlin. https://mediendienstintegration.de/fileadmin/Dateien/Expertise_Anker-Zentren_August_2018.pdf. Zugegriffen: 8. Aug. 2019.

Hinger, S., \& Schäfer, P. (2017). Wohnst Du schon - oder wirst Du noch untergebracht? In Bundeszentrale für politische Bildung (Hrsg.), Kurzdossiers „Zuwanderung, Flucht und Asyl: Aktuelle Themen“. Berlin: Bundeszentrale für politische Bildung. 
Hofmann, R., \& Scherr, A. (2017). Verwahrung in Aufnahmelagern oder Willkommenskultur? Eine Fallstudie zur Erstaufnahme von Geflüchteten, 1-13. https://ratfuermigration.files.wordpress.com/2018/ 08/vorstudie_hofmann_scherr_2017.pdf. Zugegriffen: 13. Aug. 2019.

Homfeldt, H. G. (2020). Kinder und Jugendliche mit Behinderung sind in erster Linie Kinder und Jugendliche. In K. Liel \& A.L. Rademaker (Hrsg.), Gesundheitsförderung und Prävention - Quo vadis Kinder- und Jugendhilfe? (S. 147-156). Weinheim und Basel: Beltz Juventa.

Horn, V., \& Schweppe, C. (2019). Gewaltpotentiale und Gewaltschutz in Privathaushalten mit 24-StundenPflegekräften. Explorative Annäherungen. Zeitschrift für Sozialpädagogik, 17(4), 394-413.

Initiative Hochschullehrender zu Sozialer Arbeit in Gemeinschaftsunterkünften (2016). Positionspapier: Soziale Arbeit in Gemeinschaftsunterkünften - professionelle Standards und sozialpolitische Basis. Berlin. https://www.fluechtlingssozialarbeit.de/Positionspapier_Soziale_Arbeit_mit_Gefl\%C3 $\%$ BCch teten.pdf. Zugegriffen: 23. Febr. 2020.

Innenministerium Mecklenburg-Vorpommern (2019). Minister Seehofer und Caffier unterzeichnen Vereinbarung zur effektiveren Gestaltung der Asylverfahren zwischen Bund und Land. Pressemitteilung 9. April 2019. https://www.regierung-mv.de/Landesregierung/im/Aktuell/?id=148660\& processor=processor.sa.pressemitteilung. Zugegriffen: 20. Febr. 2020.

Innenministerium Schleswig-Holstein (2019). Innenministerium Schleswig-Holstein und Bundesinnenministerium schließen Vereinbarung für Aufbau und Betrieb eines Kompetenzzentrums für Ankunft, Verteilung und Rückkehr. Pressemitteilung 13. Juni 2019. https://schleswig-holstein.de/DE/ Landesregierung/IV/Presse/PI/2019/190613_VereinbarungKompetenzzentrum.html. Zugegriffen: 21. Febr. 2020.

Jensen, O. B. (2009). Flows of meaning, cultures of movements - urban mobility as meaningful everyday life practice. Mobilities, 4(1), 139-158.

Kewes, A. (2016). Urban Citizenship - Oder: Über den Versuch, dem „System“ auf Augenhöhe zu begegnen. In S. Rother (Hrsg.), Migration und Demokratie (S. 139-160). Wiesbaden: Springer.

Koalitionsvertrag zwischen CDU, CSU und SPD (2018). Ein neuer Aufbruch für Europa. Eine neue Dynamik für Deutschland. Ein neuer Zusammenhalt für unser Land. 19. Legislaturperiode. Berlin: Koalitionsvertrag zwischen CDU, CSU und SPD.

Krause, U. (2018). Gewalterfahrungen von Geflüchteten. State-of-Research Papier 03. Osnabrück: IMIS und Bonn: BICC. https://flucht-forschung-transfer.de/wp-content/uploads/2017/05/Stateof-Research-03-Gewalterfahrungen-von-Flüchtlingen-Ulrike-Krause-1.pdf. Zugegriffen: 21. Febr. 2020.

Kreichauf, R. (2018). From forced migration to forced arrival: the campization of refugee accommodation in European cities. Comparative Migration Studies, 6(7), 1-22. https://doi.org/10.1186/s40878-0170069-8.

Lahusen, C., \& Schneider, S. (2017). Asyl verwalten. Eine Einleitung. In C. Lahusen, \& S. Schneider (Hrsg), Asyl verwalten. Zur bürokratischen Bearbeitung eines gesellschaftlichen Problems (S. 7-24). Bielefeld: transcript.

Loick, D. (2017). Wir Flüchtlinge. Überlegungen zu einer Bürgerschaft jenseits des Nationalstaates. Leviathan, 45(4), 1-18.

Muy, S. (2019). Die Gegenwart der Lager - revisited. Überlegungen zu „AnKER“-Zentren und Sozialer Arbeit. In C. Resch (Hrsg.), Migration als soziale Praxis: Kämpfe um Autonomie und repressive Erfahrungen (S. 190-205). Münster: Westfälisches Dampfboot.

Nail, T. (2019). Being and motion. New York: Oxford University Press.

Oevermann, U. (2002). Klinische Soziologie auf der Basis der Methodologie der objektiven Hermeneutik. http://www.ihsk.de/publikationen/Ulrich_Oevermann-Manifest_der_objektiv_hermeneutischen_ Sozialforschung.pdf. Zugegriffen: 12. Aug. 2019.

Pieper, T. (2013). Die Gegenwart der Lager. Zur Mikrophysik der Herrschaft in der deutschen Flüchtlingspolitik (2. Aufl.). Münster: Westfälisches Dampfboot.

Pürckhauer, A. (2019). Was wissen wir über „AnKER-Zentren“? Mediendienst Migration. https:// mediendienst-integration.de/de/artikel/was-wissen-wir-ueber-anker-zentren.html. Zugegriffen: 21. Febr. 2020.

Retkowski, A., Treibel, A., \& Tuider, E. (Hrsg.). (2018). Handbuch Sexualisierte Gewalt und pädagogische Kontexte. Weinheim und Basel: Beltz Juventa.

Rölli, M., \& Nigro, R. (2017). Einleitung. In M. Rölli \& R. Nigro (Hrsg.), Vierzig Jahre „Überwachen und Strafen “. Zur Aktualität der Foucault'schen Machtanalyse (S. 7-20). Bielefeld: transcript.

Salazar, N.B. (2018). Theorizing mobililty through concept and figures. Tempo Social. Revista de Sociologia da USP, 30(2), 153-168. 
Schader, M., Rohmann, T., \& Münch, S. (2018). Isolation im Gesetz verankern? Zu den Plänen der großen Koalition, zentrale Aufnahme-, Entscheidungs- und Rückführungseinrichtungen einzuführen. Zeitschrift für Flüchtlingsforschung, 2(1), 91-107.

Schäfer, P. (2015). Das Flüchtlingswohnheim. Raumcharakter und Raumpraxis in der Gemeinschaftsunterkunft. sinnprovinz. kultursoziologische working papers, 7, 1-10.

Scherf, M. (2009). Objektive Hermeneutik. In S. Kühl, P. Strodtholz \& A. Teffertshofer (Hrsg.), Handbuch Methoden der Organisationsforschung. Quantitative und Qualitative Methoden (S. 300-325). Wiesbaden: VS.

Schieß1, S. (2018). AnKER-Zentren: „Normalfall“ Lager? Die Institutionalisierung der Abgrenzung. In T. Müller-Heidelberg, M. Pelzer, M. Heiming, C. Röhner, R. Gössner \& M. Fahrner (Hrsg.), Grundrechte-Report 2018. Zur Lage der Bürger- und Menschenrechte in Deutschland (S. 16-19). Frankfurt am Main: Fischer.

Schittenhelm, K. (2015). Asylsuchende im Blickfeld der Behörde. Explizites und implizites Wissen in der Herstellung von Asylbescheiden in Deutschland. Soziale Probleme, 26, 137-150.

Schröer, W., \& Wolff, S. (2018). Sozialpädagogik und Organisationspädagogik. In M. Göhlich, A. Schröer \& S. M. Weber (Hrsg.), Handbuch Organisationspädagogik (S. 59-70). Wiesbaden: VS.

Täubig, V. (2009). Totale Institution Asyl. Empirische Befunde zu alltäglichen Lebensfïhrungen in der organisierten Desintegration. Weinheim: Juventa.

Thiel, J., \& Jahr, C. (2017). Begriff und Geschichte des Lagers. http://www.bpb.de/gesellschaft/migration/ kurzdossiers/246175/begriff-und-geschichte-des-lagers?p=all. Zugegriffen: 8. Aug. 2019.

Wihstutz, A. (Hrsg.). (2019). Zwischen Sandkasten und Abschiebung. Zum Alltag junger Kinder in Unterkünften für Geflüchtete. Opladen, Berlin und Toronto: Barbara Budrich.

Wolff, S. (2008). Dokumenten- und Aktenanalyse. In U. Flick, E. von Kardoff \& I. Steinke (Hrsg.), Qualitative Forschung. Ein Handbuch (S. 502-513). Reinbek: Rowohlt.

Zeller, M., Köngeter, S., \& Meier, L. (2020). Vertrauen, Partizipation und Agency. Vertrauen und Zukunftsvorstellungen bei jungen Geflüchteten im Übergang. In S. Göbel, U. Karl, M. Lunz, U. Peters \& M. Zeller (Hrsg.), Wege junger Menschen aus Heimen und Pflegefamilien (S. 204-224). Weinheim und Basel: Beltz Juventa. 